\title{
Synchronous multiple primary lung cancer: An increasing clinical occurrence requiring multidisciplinary management
}

Delphine Trousse, MD, ${ }^{\text {a }}$ Fabrice Barlesi, MD, ${ }^{\mathrm{b}}$ Anderson Loundou, $\mathrm{PhD},{ }^{\mathrm{c}}$ Anne Marie Tasei, MD, ${ }^{\mathrm{d}}$ Christophe Doddoli, MD, ${ }^{a}$ Roger Giudicelli, MD, ${ }^{a}$ Philippe Astoul, MD, PhD, ${ }^{\text {b }}$ Pierre Fuentes, MD, ${ }^{\text {a }}$ and Pascal Thomas, MD, FECTS ${ }^{a}$

Earn CME credits at http://cme.ctsnetjournals.org
From the Departments of Thoracic Surgery, ${ }^{\mathrm{a}}$ Thoracic Oncology, ${ }^{\mathrm{b}}$ and Pathology, ${ }^{\mathrm{d}}$ Sainte Marguerite University Hospital; and Department of Public Health and Biostatistics, ${ }^{\mathrm{c}}$ Faculty of Medicine, Université de la Méditerranée (Aix-Marseille II) and Assistance Publique-Hôpitaux de Marseille, Marseille, France.

Received for publication Sept 29, 2006; revisions received Dec 16, 2006; accepted for publication Jan 5, 2007.

Address for reprints: Pascal Thomas, MD, FECTS, Department of Thoracic Surgery, Ste Marguerite Hospital-CHU Sud, 270 Bd Ste Marguerite, 13274 Marseille Cedex 9, France (E-mail: pathomas@ap-hm.fr).

J Thorac Cardiovasc Surg 2007;133:1193-200 $0022-5223 / \$ 32.00$

Copyright (C) 2007 by The American Association for Thoracic Surgery

doi:10.1016/j.jtcvs.2007.01.012
Objective: No guidelines detailing recommendations for the selection and treatment of patients with synchronous multiple primary lung cancer have been published. We report on a single-institution experience with synchronous multiple primary lung cancer, with emphasis on long-term survival.

Methods: We performed a retrospective study of 125 consecutive patients with synchronous multiple primary lung cancer who underwent operation between 1985 and 2006. Various treatment strategies were applied, including perioperative therapy. Potential prognosticators were submitted to univariate and multivariate analyses.

Results: Tumors were bilateral $(\mathrm{n}=34)$ or ipsilateral $(\mathrm{n}=91)$. Optimal surgical treatment (complete anatomic resection with radical lymphadenectomy) was possible in $65.6 \%$ of the cases. pN0 disease was present in $32.3 \%$ of the patients; 30-day and 90 -day mortality rates were $4.5 \%$ and $11 \%$, respectively. Two- and 5-year overall survivals were $61.6 \%$ and $34 \%$, respectively, with a median survival of 35 months. On univariate analysis, smoking status, high Charlson index, low forced expiratory volume in 1 second, occurrence of postoperative complications, and performance of a pneumonectomy affected the overall survival adversely. Conversely, bilateral disease, location in the same lobe, and $\mathrm{pNO}$ disease were favorable prognosticators. On multivariate analysis, low forced expiratory volume in $1 \mathrm{sec}-$ ond, nonoptimal surgical treatment, and performance of a pneumonectomy were independent predictors of poor long-term survival, whereas female sex, younger age, asymptomatic disease, $\mathrm{pN} 0$ status, and performance of an adjuvant treatment affected the survival favorably.

Conclusions: Provided there is an appropriate selection process, patients with synchronous multiple primary lung cancer are expected to benefit from surgery. Optimal surgery should be performed, but pneumonectomy should be avoided whenever possible. Adjuvant treatment is suggested to provide an added survival advantage.

$\mathrm{S}$ ynchronous multiple primary lung cancer (MPLC) is a presumed uncommon entity, but its true incidence, ranging from $0.2 \%$ to $20 \%,{ }^{1}$ is increasing as the result of the widespread use of early detection tools such as multislice spiral computed tomography (CT), fluorescence endoscopy, and positron emission tomography (PET) scanning. However, simultaneous tumors are ambiguously assessed by the TNM staging system, ${ }^{2}$ which classifies this clinical situation either as a locally advanced disease when both tumors are located in a same lobe or as a metastatic tumor in the other cases. In the absence of easily available genetic or molecular markers, differentiation between MPLC and isolated pulmonary metastasis will 


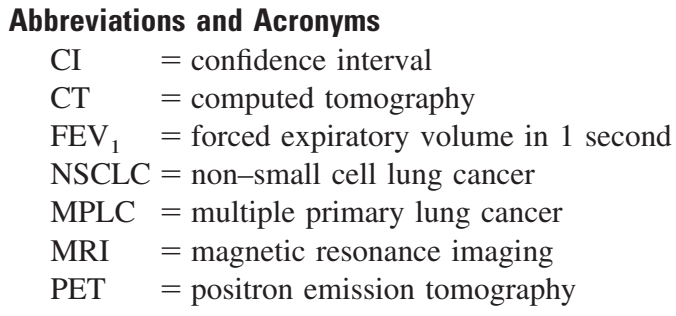

remain difficult in the clinical setting, leading to controversies regarding management considerations. There are currently no published guidelines detailing recommendations for the selection and treatment of such patients. In a Manichean plan, two opposite strategies are faced: the belief that any resectable disease should be resected and the belief that surgery is a futile treatment for a potentially metastatic situation. From a pragmatic point of view, a multifocal disease in the same lobe does not change the type of surgery to be applied in most instances. Clearly, this is not the case when a pneumonectomy or a bilateral lung resection is required, showing evidence of some limitations regarding the cardiopulmonary reserve. Conversely, patients with non-small cell lung cancer (NSCLC) have a low chance to date of being cured by chemotherapy alone at any stage.

As proponents of a surgical approach within a multidisciplinary strategy, we aimed to report on our experience with synchronous MPLC, with emphasis on long-term survival.

\section{Materials and Methods}

Between July 1985 and May 2006, 125 consecutive patients underwent operation with synchronous MPLC in a single academic thoracic surgery department among 2785 patients treated for a primary lung cancer during the period. Patients were identified by screening a database in which any patient with an NSCLC was entered prospectively. Patients with typical carcinoid tumors or pure bronchioloalveolar adenocarcinomas were not included in the present study. Tumors were defined as synchronous MPLC when multiple locations were discovered simultaneously $(\mathrm{n}=112)$ or within a 24-month delay $(\mathrm{n}=13)$, provided that the second tumor was ascertained retrospectively to be present at the time of the treatment of the first tumor by reviewing the imaging files. Tumors were defined as MPLC when histology was different, when tumors were located in different segments of the same lobe, in different lobes, or bilaterally, and in the absence of evidence of an extrathoracic disease. Tumors with satellite nodules $(n=84)$, defined as smaller tumors (diameter $<1 \mathrm{~cm}$ ) located close to the original cancer, in the same segment were not included in the study.

During the same period, 54 patients with metachronous primary lung cancer were treated surgically at our institution.

\section{Patients}

There were 27 women $(21.6 \%)$ and 98 men (78.4\%) aged from 40 to 80 years. The median age was 61.5 years ( \pm 9.9 years). There were 91 patients $(72.8 \%)$ with unilateral synchronous MPLC and 34 patients $(27.2 \%)$ with bilateral synchronous MPLC. The American Society of Anesthesiologists score was calculated for all patients on the basis of their preoperative physical examination results. The mean score was $2 \pm 0.8$, ranging from $1(\mathrm{n}=41)$ to $4(\mathrm{n}=2)$.

A total of 111 patients $(88 \%)$ were smokers, and 14 patients (11\%) were nonsmokers. Eighty patients $(63 \%)$ smoked more than 40 packs/year. At the time of surgery, 60 patients (48\%) were current smokers.

Nineteen patients (15\%) previously had an additional extrathoracic malignancy (mean delay: 75.2 months) that was considered in complete remission. Seven of them had a history of oropharyngeal carcinomas, and 4 of them had a history of lymphoma. The 8 other patients were treated for various primary tumors.

Thirty-six patients $(28 \%)$ had cardiovascular comorbidities. Chronic obstructive pulmonary disease was present in 48 patients (38\%), according to the Global Initiative for Chronic Obstructive Lung Disease guidelines. The mean Charlson score was $2 \pm 1.7$, ranging from $0(n=11)$ to $9(n=1)$.

The disease was discovered fortuitously $(48.8 \%)$ or revealed by symptoms $(51.2 \%)$.

\section{Preoperative Workup}

All patients underwent chest radiography, bronchoscopy, and chest CT scan including the upper abdomen (basic workup). An abnormal bronchoscopy was present in 33 cases $(26.4 \%)$, and in 34 patients $(27.2 \%)$ the histologic type of the tumors was known before surgery by the means of bronchoscopy or CT scan-guided biopsies. The majority of patients $(n=116 ; 93 \%)$ underwent brain CT scan. One supplementary patient underwent brain magnetic resonance imaging (MRI). Routine bone scintigraphy was performed in 53 patients $(42.4 \%)$. The last 12 patients of this series were explored with 18-fluorodeoxyglucose PET scanning (9.6\%). Invasive staging was performed in 7 patients with bilateral synchronous MPLC; 5 (4\%) underwent mediastinoscopy and 2 underwent a thoracoscopy (1.6\%). Extensive preoperative workup was defined by the additional performance of routine brain CT scan or MRI, and bone scintigraphy or PET scanning to the basic workup. Such extensive preoperative workup was performed in 68 patients (54.4\%): 27 patients $(79.4 \%)$ in the bilateral group and 41 patients $(45 \%)$ in the unilateral group. The treatment strategy was decided at the occasion of the institutional oncologic multidisciplinary meeting.

\section{Surgery}

Whenever possible, an anatomic resection (ie, lobectomy or pneumonectomy) was performed for the 2 tumors, together with an ipsilateral lymphadenectomy, and was considered as an optimal surgery. Sublobar resections (ie, wedge or segmentectomy) and lymph node sampling were regarded as nonoptimal procedures. Lymphadenectomy was defined by the harvesting of at least 10 lymph nodes and the removal of two or more ipsilateral mediastinal stations. ${ }^{3}$ When both criteria were not satisfied at the reading of the pathology report, the $\mathrm{N}$ status was graded as $\mathrm{pNx}$.

Neoadjuvant treatment included any chemotherapy regimen given before surgery or between the two surgical sessions, when applicable in case of bilateral disease. 


\section{Pathologic Staging}

Pathologic staging was based on the International System for Staging Lung Cancer. ${ }^{2}$ A total of 123 patients had 2 cancers (unilateral tumors in 89 and bilateral tumors in 34). Among the unilateral group, 62 tumors were in the same lobe and 27 tumors were in a different lobe. Two patients had 3 unilateral lung carcinomas, in the same lobe in 1 patient and in a different lobe in 2 patients.

Histology was identical in 104 cases (83.2\%). Adenocarcinoma and squamous cell carcinoma were the predominant histologic types, $52 \%$ and $28.8 \%$, respectively, in this group. Histology was different in $(\mathrm{n}=12)$ in the bilateral group and $(\mathrm{n}=9)$ unilateral group. Large cell carcinomas accounted for $8.8 \%$ of the cases.

There were 62 patients $(49.6 \%$ ) classified with pM1 pulmonary and 63 patients $(50.4 \%)$ classified with pT4 for multifocal tumors in the same lobe. ${ }^{2}$ In the group classified with pM1 pulmonary, 28 patients $(45.2 \%)$ had ipsilateral cancers in a different lobe and 34 patients $(54.8 \%)$ had bilateral cancers. Among patients with bilateral cancers, $11(32.3 \%)$ had $\mathrm{pN} 0$ for 1 side and $\mathrm{pNx}$ for the other side, $5(14.7 \%)$ had at least 1 side with lymph node invasion $(\mathrm{pN}+), 11(32.3 \%)$ had pN0 bilaterally, and 7 (20.6\%) had pNx for both sides. Among the ipsilateral group, 33 patients (36.2\% of this group) had a pN0 disease, 32 patients $(35.2 \%)$ had a pN2 disease, 17 patients $(18.7 \%)$ had a pN1 disease, and 9 patients $(9.9 \%)$ had a $\mathrm{pNx}$ disease.

\section{Statistics}

The statistical analysis was performed by using the Statistical Package for the Social Sciences version 13.0 software package (SPSS Inc, Chicago, Ill). Quantitative variables were expressed as mean \pm standard deviation. For all patients, survival was calculated from the date of surgery until the date of last follow-up if the patient was still alive on May 31, 2006, or the date of death collected by consulting the City Hall registry. Survivorship was calculated according to the Kaplan-Meier method, including the operative mortality. Differences in survival were tested for significance by the log-rank test. A Cox proportional hazards model was fit to examine and adjust for any explanatory variables. Forward stepwise procedure was used to select the variables with the greatest prognostic value $(P<.05)$.

\section{Results}

\section{Surgery}

Three patients with a bilateral disease had only one side surgically explored because of substantial postoperative complication or insufficient pulmonary reserve preventing a bilateral resection. An additive treatment (chemotherapy and/or radiotherapy) was performed in these 3 cases. Among the 31 patients with a bilateral disease who underwent operation on both sides, 2 were treated during the same session and 29 were treated sequentially with a median delay of 5.7 months.

The details of the surgical procedures are given in Table 1. Radical lymphadenectomy was performed in 94 patients (75.2\%), and lymph node sampling was performed in 31 patients $(24.8 \%)$. A mean of $3.5(1-8)$ nodal stations and 13.3 ( \pm 11.4$)$ lymph nodes were explored at first surgery,
TABLE 1. Surgical procedures for synchronous multiple primary lung cancer ( $n=125$ patients)

\begin{tabular}{lcc}
\hline & No. of & \\
& patients & $\%$ \\
\hline Surgical procedure for unilateral MPLC & 91 & 72.8 \\
Sublobar resection & 14 & 11.2 \\
(Bi)lobectomy & 34 & 27.2 \\
Extended lobectomy* & 3 & 2.4 \\
Pneumonectomy & 40 & 32 \\
Surgical procedure for bilateral MPLC & 34 & 27.2 \\
Sublobar and abstention & 1 & 0.8 \\
Lobectomy and abstention & 2 & 1.6 \\
Bilateral sublobar resection & 6 & 4.8 \\
Lobectomy and sublobar resection & 14 & 11.2 \\
Extended lobectomy and sublobar resection & 1 & 0.8 \\
Bilateral lobectomy & 7 & 5.6 \\
Extended lobectomy and lobectomy & 2 & 1.6 \\
Pneumonectomy and sublobar resection & 1 & 0.8 \\
\hline
\end{tabular}

*Extended lobectomy : bronchial and/or vascular sleeve lobectomy.

and $2.7(1-11)$ nodal stations and $8.3( \pm 9.2)$ lymph nodes were explored at the second surgery. In total, 82 patients (65.6\%) underwent an optimal surgical treatment and 43 patients $(34.4 \%)$ received limited resections because of the presence of comorbidity or poor performance status.

\section{Treatment Strategy}

Sixty-three patients (50.4\%) underwent surgery alone.

Neoadjuvant platin-based chemotherapy was applied in 19 patients (15.2\%): 2 cycles in 13 patients, 3 cycles in 2 patients, and 4 cycles in 4 patients.

Adjuvant treatment consisted of chemotherapy or radiotherapy or both and was administered to 62 patients (49.6\%). Fifty-six patients (44.8\%) received 2 to 5 sessions of adjuvant platin-based chemotherapy (mean: 3 sessions). Twenty-three patients (18.4\%) underwent adjuvant radiotherapy. Eighteen patients (14.4\%) underwent chemoradiotherapy. Three patients $(2.4 \%)$ who died after surgery could not receive the foreseen adjuvant treatment. Finally, 66 patients $(52.8 \%)$ received a local treatment only (surgery possibly associated with radiotherapy), and 59 patients $(47.2 \%)$ received an additional systemic treatment.

\section{Postoperative Course and Long-term Survival}

Thirty-four patients $(27.2 \%)$ had postoperative complications. Sputum retention was the most frequent (8.8\%), followed by pneumonia (6.4\%) and bronchial fistula (1.6\%). The 30-day mortality rate $(\mathrm{n}=6)$ was $4.5 \%(95 \%$ confidence interval $[\mathrm{CI}]: 1.75-9.9)$. Causes of death were bronchial fistula, acute respiratory distress syndrome, cardiac failure, and multiple organ failure. The overall 90-day mortality rate $(\mathrm{n}=14)$ was $11 \%(95 \%$ CI: 6.16-17.8). Mean hospitalization stay was 17 days. 


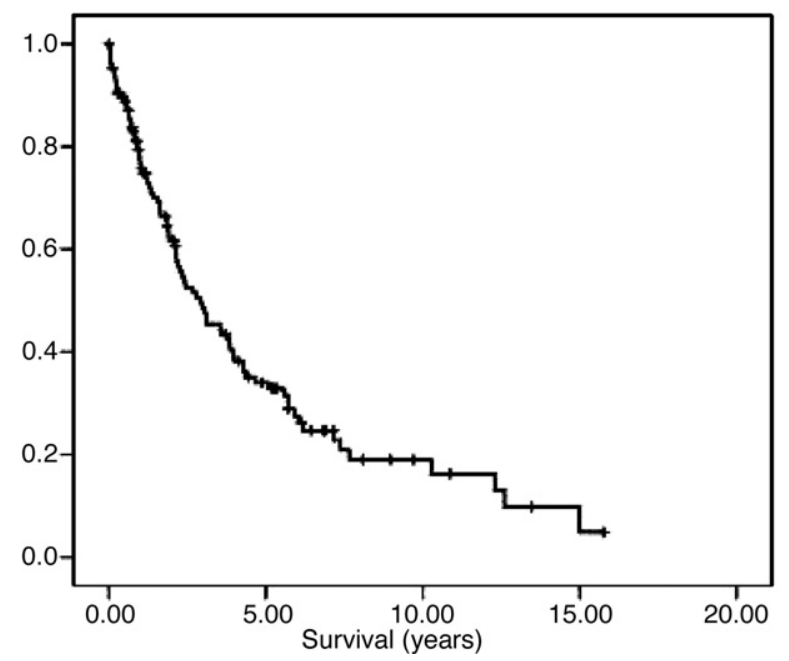

Figure 1. Overall survival of 125 patients with synchronous MPLC. MPLC, multiple primary lung cancer.

Mean duration of follow-up was 57.2 months (95\% CI: 45.2-69.2). Fourteen patients (11.2\%) were lost to followup. At last follow-up, 83 patients $(66.4 \%)$ were dead and 42 patients $(33.6 \%)$ were still alive. The overall 2- and 5-year survivals were $61.6 \%$ (95\% CI: 52.6-70.6) and 34\% (95\% CI: 24.8-43.2), respectively. Median survival was 34.9 months (95\% CI: 21.7-48.1). The overall 10-year survival was 19\% (95\% CI: 10.1-27.9) (Figure 1).

Forty-nine patients $(38.6 \%)$ experienced recurrence within a mean delay of 9.3 months (median: 4.4 months). The lung was the site of first recurrence in $40.8 \%$ of the cases, followed by multifocal $(30.6 \%)$, brain $(22.4 \%)$, bone $(4.1 \%)$, and liver $(2 \%)$ sites. Disease-free survivals were $60 \%$ (95\% CI: $50.1-70.1)$ at 2 years, $56 \%$ (95\% CI: $45.2-$ $66.8)$ at 5 years, and $51 \%(95 \%$ CI: $38.1-64.6)$ at 10 years.
No significant difference in 5-year survival was observed between patients who received a local treatment only and patients who also received chemotherapy $(52.8 \%$ vs $47.2 \%$, $P=.58)$.

On univariate analysis, patients with a bilateral disease had a better survival than those with a unilateral disease $(P=.025)$. Unilateral disease in the same lobe was also associated with a better outcome than in a different lobe $(P=.033)$ (Figure 2$)$. The following continuous variables appeared as prognostic parameters (Table 2): Charlson index, forced expiratory volume in 1 second $\left(\mathrm{FEV}_{1}\right)$ (percentage), and $\mathrm{FEV}_{1}$ /forced vital capacity (percentage). Current smokers had an impaired survival, as well as patients who underwent a pneumonectomy. The lymph node status significantly affected survival: The best overall survival was observed for patients with a pN0 disease (51.4\%; 95\% CI: 16.6-86.1) compared with those with a $\mathrm{pN}+$ disease $(15.4 \% ; 95 \% \mathrm{CI}: 7.8-23.1)$, whereas patients with a pNx disease $(42.5 \%$; $95 \%$ CI: $28.3-56.8)$ had an intermediate prognosis $(P=.01)$ (Figure 3$)$.

Cox's multivariate regression model identified the following variables as independent adverse prognosticators of overall survival (Table 3): male sex, patient age more than 60 years, symptomatic disease, low $\mathrm{FEV}_{1}(\%)$, performance of a pneumonectomy, absence of an adjuvant therapy, absence of an optimal surgical resection, and presence of lymph node metastasis. Table 4 details the variables that did not enter the model at the last step of the analysis.

\section{Discussion}

According to the literature, MPLC occurs with an incidence of $0.2 \%$ to $20 \%,{ }^{1,4-6}$ when referring to the definition criteria of Martini and Melamed. ${ }^{7}$ However, these criteria are not easily applicable in the case of synchronous MPLC, a clinical entity not recognized by the International Union Against Cancer TNM classification, which scales this con-

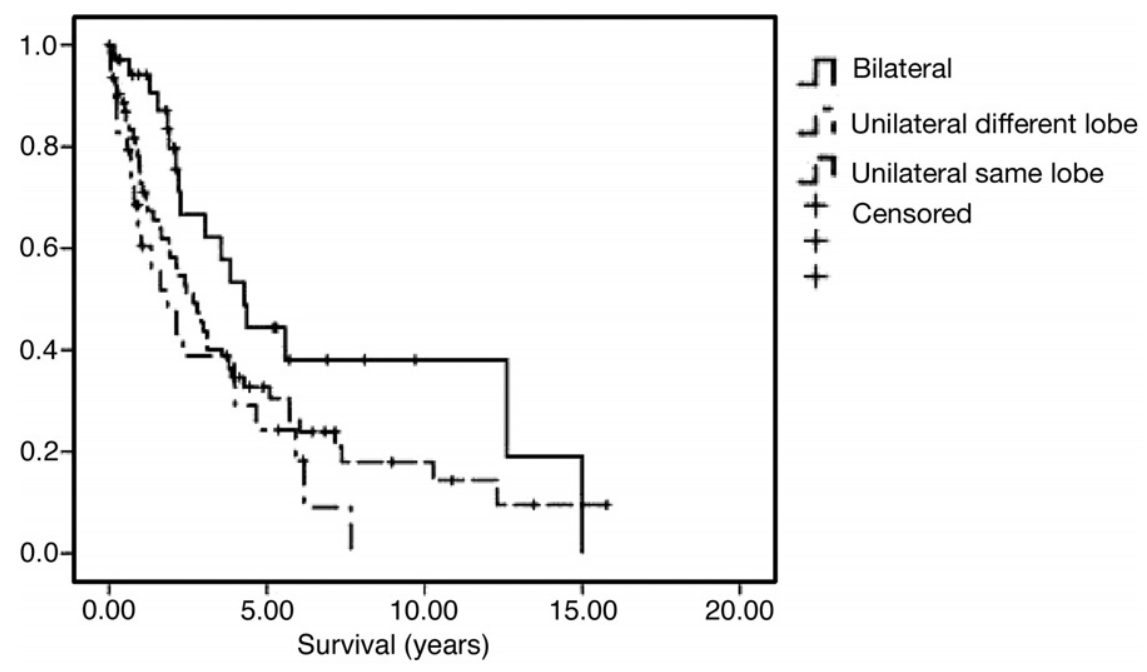

Figure 2. Overall survival according to tumor location. 
TABLE 2. Results of a univariate analysis for prognostic factors negatively affecting overall survival in 125 patients with synchronous multiple primary lung cancer

\begin{tabular}{|c|c|c|}
\hline Variables & No. & $P$ value \\
\hline $\operatorname{Sex}(M / F)$ & $98 / 27$ & .30 \\
\hline Age $( \pm 60$ y) & $50 / 75$ & .15 \\
\hline Bilateral vs unilateral & $34 / 91$ & .02 \\
\hline Bilateral vs same lobe vs different lobe & $34 / 63 / 28$ & .03 \\
\hline Symptomatic disease $(\mathrm{Y} / \mathrm{N})$ & $64 / 61$ & .11 \\
\hline Past/current smokers & $65 / 60$ & .01 \\
\hline Tobacco ( \pm 40 packs/y) & $40 / 85$ & .74 \\
\hline ASA score (1 and 2 vs 3 and 4$)$ & $89 / 36$ & .32 \\
\hline NYHA classification (1 and 2 vs 3 ) & $109 / 16$ & .17 \\
\hline COPD (Y/N) & $47 / 72$ & .09 \\
\hline $\mathrm{FEV}_{1}$ (L) & - & .12 \\
\hline FEV $_{1}(\%)$ & - & .002 \\
\hline $\mathrm{FEV}_{1} / \mathrm{FVC}(\%)$ & - & .008 \\
\hline Cardiovascular comorbidity (Y/N) & $35 / 90$ & .83 \\
\hline History of other cancers (Y/N) & $18 / 107$ & .72 \\
\hline Charlson index & - & .05 \\
\hline Extensive preoperative workup (Y/N) & $66 / 59$ & .23 \\
\hline Preoperative histology $(\mathrm{Y} / \mathrm{N})$ & $33 / 92$ & .22 \\
\hline Optimal surgery $(\mathrm{Y} / \mathrm{N})$ & $82 / 43$ & .34 \\
\hline Lymphadenectomy (Y/N) & $94 / 31$ & .41 \\
\hline Pneumonectomy (Y/N) & $41 / 89$ & $<10^{-4}$ \\
\hline Different histology (Y/N) & $21 / 104$ & .82 \\
\hline ADK vs other histology & $64 / 61$ & .36 \\
\hline T4 vs M1 & $63 / 62$ & .56 \\
\hline pNO vs pNx vs pN+ & $44 / 27 / 54$ & .01 \\
\hline pT main tumor vs $\mathrm{pT}$ other tumor(s) & & .58 \\
\hline Postoperative complications (Y/N) & $34 / 91$ & .07 \\
\hline Neoadjuvant therapy (Y/N) & $19 / 106$ & .65 \\
\hline Adjuvant treatment (Y/N) & $58 / 67$ & .43 \\
\hline Local vs systemic treatment & $66 / 59$ & .58 \\
\hline
\end{tabular}

$\overline{A S A}$, American Society of Anesthesiologists; NYHA, New York Heart Association; COPD, chronic obstructive pulmonary disease; $F E V_{1}$, forced expiratory volume in 1 second; $F V C$, forced vital capacity; $A D K$, adenocarcinoma. Statistically significant variables in bold.

dition in "nonsurgical" stages (IIIB and IV). ${ }^{8}$ With the use of more realistic criteria, the incidence of synchronous MPLC was $4.5 \%$ in our experience. Given the high prevalence of benign nodules detected at the occasion of the preoperative workup in patients with lung cancer, and the inconsistency of predictions based on CT features, there is a need for improving diagnostic accuracy of synchronous MPLC to avoid overlooking a possible curative treatment for the patient. ${ }^{9,10}$ A different histology in two obviously distinguishable lung tumors is a strong argument in favor of the primary nature of both diseases. However, this situation is rare, occurring in approximately $15 \%$ of cases, in our experience. In the case of similar histologic conditions, to differentiate a pulmonary metastasis from a true synchronous lung cancer is difficult in the absence of genetic and molecular analysis, not available in routine clinical practice.

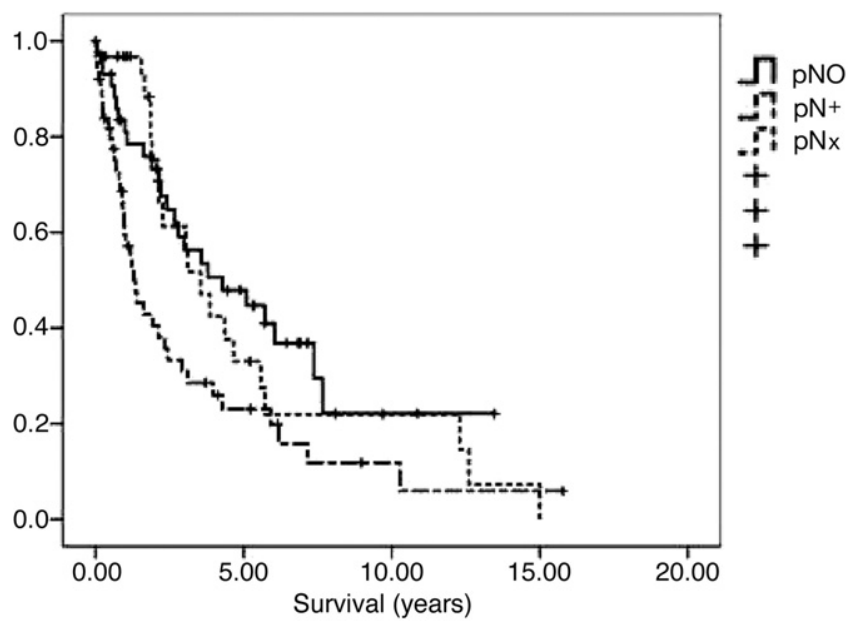

Figure 3. Overall survival according to lymph node status (pN).

It is also difficult to discriminate between synchronous MPLC and multiple lung metastases from an occult distant cancer. A definitive proof of the malignancy of all suspicious lung nodules is critical and should be obtained before defining the optimal treatment. ${ }^{11}$

Searching for distant metastases or confirming complete remission of a previous extrathoracic neoplasm is crucial. ${ }^{11-15}$ Brain MRI, fluorescence bronchoscopy, and whole-body 18-fluorodeoxyglucose PET scanning added valuable contributions to the noninvasive staging of lung cancer. The accuracy of brain MRI in diagnosing brain metastases is definitely higher than that of brain CT scan. Fluorescence bronchoscopy was proven to improve the detection of concomitant central premalignant lesions or synchronous invasive lung cancers. PET also carries higher sensitivity and specificity than does CT scanning for local regional staging, detection of occult liver or adrenal metastases, and disclosure of incidental coexistent neoplasms. We

TABLE 3. Results of a multivariate analysis for prognostic factors negatively affecting survival in 125 patients with synchronous multiple primary lung cancer

\begin{tabular}{lcccc}
\hline Variable & \multicolumn{4}{c}{ Hazard } \\
& No. & ratios & 95\% Cl & $\boldsymbol{P}$ value \\
\hline Sex (male) & 98 & 2.5 & $1.15-5.42$ & .021 \\
Age $(<60$ y) & 75 & 0.53 & $0.31-0.91$ & .022 \\
Asymptomatic (yes vs no) & 61 & 0.42 & $0.24-0.72$ & .002 \\
FEV ${ }_{1}(\%)$ & 119 & 0.95 & $0.93-0.98$ & $<.0001$ \\
Pneumonectomy (yes vs no) & 41 & 6.6 & $3.34-13.1$ & $<.0001$ \\
Optimal surgery (no vs yes) & 43 & 2.3 & $1.14-4.75$ & .02 \\
Adjuvant treatment (no vs yes) & 67 & 1.8 & $1.02-3.33$ & .043 \\
pNO disease (no vs yes) & 44 & 2.9 & $1.2-6.9$ & .004
\end{tabular}

$\mathrm{Cl}$, Confidence interval; $\mathrm{FEV}{ }_{1}$, forced expiratory volume in 1 second. 
TABLE 4. Scores at the last step of an analysis of overall survival for other relevant covariates excluded from the Cox model

\begin{tabular}{llc}
\hline Variables & Scores & $\boldsymbol{P}$ value \\
\hline Tobacco $<40$ packs/y & 0.58 & .45 \\
NYHA (1 and 2 vs 3) & 2.07 & .15 \\
Absence of cardiovascular comorbidity & 0.05 & .82 \\
FEV $/$ FVC (\%) & 0.008 & .93 \\
Absence of COPD & 0.016 & .90 \\
Charlson index & 0.30 & .58 \\
Absence of extensive preoperative workup & 0.008 & .93 \\
Absence of neoadjuvant therapy & 0.03 & .86 \\
Bilateral vs same lobe vs different lobe & 0.98 & .61 \\
pT status & 2.40 & .12 \\
Absence of postoperative complications & 0.23 & .63
\end{tabular}

COPD, Chronic obstructive pulmonary disease; $F E V_{1}$, forced expiratory volume in 1 second; FVC, forced vital capacity; NYHA, New York Heart Association.

failed to ascribe any prognostic impact to an "extensive" preoperative workup in comparison with a "basic" workup, because modalities of such extensive workup changed along the period according to evolving accessible technologies. An extensive preoperative workup was performed in more than three-fourths of the patients with bilateral synchronous MPLC, but in less than one-half of patients with unilateral synchronous MPLC. Accordingly, invasive preoperative staging was performed only in the former subset of patients to rule out mediastinal lymph node metastasis. ${ }^{9,11}$ The bilateral location of the disease was identified as a favorable prognosticator on univariate analysis.

Bilateral MPLC is usually approached through two distinct thoracotomies, even if sternotomy may offer an adequate exposure for lung resections and extended bilateral mediastinal lymphadenectomy. ${ }^{16-24}$ In the majority of our patients, we favored a staged bilateral thoracotomy to reduce postoperative morbidity. ${ }^{23}$ In the eventuality of bilateral tumors of markedly different stages, we favored the treatment of the earliest tumor first, which usually required the lesser lung sacrifice, to preserve the feasibility and safety of a contralateral resection. This sequence may also be chosen when the histology of the smallest tumor is unknown and/or its primary nature remains uncertain. In that case, a limited resection is performed as a staging procedure, usually by video-assisted thoracoscopy. Limited resection on a single lung after contralateral pneumonectomy was performed in 1 patient. However, this situation is not comfortable technically and may result in severe complications-thus, it is not recommended.

Our results clearly show that performance of a pneumonectomy had a major adverse and independent impact on survival. The risk of death is well characterized by the attached 6.6 hazard ratio. Explanations are multiple: en- hanced postoperative morbidity and mortality, impaired cardiopulmonary reserve, and higher staged disease. Of note, such a deleterious effect of pneumonectomy has been already reported for early ${ }^{12-14}$ and advanced stages. ${ }^{15}$

Consequently, pneumonectomy should probably be avoided whenever possible, even for ipsilateral tumors located in different lobes. Alternative lung-sparing resections are also to be considered in the light of the residual pulmonary function, because, as we disclosed, $\mathrm{FEV}_{1}$ percentage is an independent prognosticator of long-term survival.

As previously suggested,${ }^{18}$ the overall prognosis of our patients with synchronous MPLC was poorer than that of those with a localized disease. To date, no standard treatment exists and few guidelines focus on the management of synchronous MPLC. ${ }^{16}$ We took great care to reduce possible bias in interpreting the results: exclusion of histologic types associated with a favorable behavior, that is, typical carcinoid and pure bronchioloalveolar adenocarcinoma, and exclusion of tumors with satellite nodules whose prognosis is now well defined. Conversely, we included those patients with multiple tumors and invaded nodes in common lymphatic pathways to comply with real-life patients, contrary to one of the criteria from Martini and Melamed. ${ }^{7}$ This last point is of paramount importance because the $\mathrm{pN}+$ status heavily influences the final results, as we showed in the present study (hazard ratio of 2.9) and as reported by others. ${ }^{16-18}$ Indeed, most of the published articles on the topic included many of these possible causes of confusion. ${ }^{1,4,10,17-20}$ We observed 5- and 10-year overall survivals of $34 \%$ and $19 \%$, respectively, that compare favorably with published data $(0 \%-33 \%$ and $0 \%-14 \%$, respectively). ${ }^{1,6,10,21-24}$ Survival was also far better than that of a metastatic disease: $10 \%$ to $23 \%$ 5-year survival for patients with a resectable NSCLC and a single synchronous brain or adrenal metastasis managed surgically, in keeping with the most optimistic reports. ${ }^{16}$ Finally, the gap we observed between overall survival and diseasefree survival also shows that non-cancer-related deaths weighed considerably in the outcome of our patients. Consequently, we found a prognostic significance to the Charlson index on univariate analysis, as did other authors in publications involving earlier stages. ${ }^{25}$

Some powerful prognostic variables on univariate analysis lost their significance at multivariate analysis. The better survival observed in patients with a bilateral disease may be partly explained by a selection bias because of a more extensive preoperative workup to rule out any extrathoracic disease and identify fit candidates for a bilateral surgery. The worse survival observed in those patients with multiple ipsilateral tumors in different lobes might reflect the fact that they underwent a pneumonectomy in most instances. The intermediate survival noted in patients with 
multifocal tumors in the same lobe is probably the end result of a balanced risk/benefit ratio in which the risks of a surgical management were not increased when compared with those of a localized disease because the type of surgery to be applied did not differ, and the long-term outcome was impaired because some of those situations included true metastatic conditions.

Overall long-term outcome was affected individually by variables linked to the patient, the disease, and the treatment. The present study adds new information on the topic, because we have identified some independent prognostic characteristics that may improve the management of such patients. Optimal surgery was defined in line with evidencebased surgical principles for the treatment of early-stage NSCLC, according to which at least a lobectomy and mediastinal lymphadenectomy should be performed. ${ }^{26}$ Our results strongly suggest that such principles should also be applied in the context of synchronous MPLC. A clear limitation, however, is the functional reserve of surgical candidates, especially when tumors are located in different lobes or lungs. However, there is growing evidence that an anatomic segmentectomy together with mediastinal lymphadenectomy may provide long-term survival similar to that associated with lobectomy in patients with peripheral tumors of less than $3 \mathrm{~cm}$ of greater size, at a lower risk, and with a lower functional impairment. ${ }^{27}$

We failed to identify any obvious divergence of outcome in patients having received any form of systemic treatment in addition to surgery when compared with that of those who received a local treatment only-that is, surgery or surgery plus adjuvant radiotherapy. One may point out that too many treatment options and drug regimens were applied during the period, precluding sufficiently sized groups for comparison. In addition, the accumulated toxicity of neoadjuvant chemotherapy and surgery, particularly when a bilateral surgery was required, might have resulted in substantial morbidity and inability of the patient to tolerate a full course of chemotherapy postoperatively. Nevertheless, approximately $40 \%$ of the patients in the present series could receive 3 cycles of adjuvant platin-based chemotherapy, and this strategy was identified as an independent predictor of improved survival by multivariate analysis. This observation is in agreement with current recommendations for the treatment of early-stage NSCLC based on meta-analysis of randomized studies that demonstrated a survival benefit of adjuvant chemotherapy in resected stages II to IIIA NSCLC. ${ }^{28}$

\section{Conclusions}

The present study demonstrates that synchronous MPLC is an increasing clinical reality, the prognosis of which seems worse than that of a localized disease but far better than that of an advanced disease. The pretreatment workup of patients with multiple lung tumors should exclude any ex- trathoracic disease and select fit candidates for a multidisciplinary management. Provided that prerequisite is met, the hypothesis of synchronous MPLC should prevail on that of a metastatic disease, and, in turn, surgery should be kept in the treatment plan. Accordingly, a lobectomy associated with lymphadenectomy and followed by adjuvant chemotherapy seems to confer substantial long-term survival at a low risk. Pneumonectomy should be avoided, and alternative lung-sparing resections should be considered whenever possible. This singular condition typifies the need for multidisciplinary meetings defining the management of any individual patient.

\section{References}

1. Rea F, Zuin A, Callegaro D, et al. Surgical results for multiple primary-lung cancers. Eur J Cardiothorac Surg. 2001;20:489-95.

2. Mountain CF. Revisions in the International System for Staging Lung Cancer. Chest. 1997;111:1710-7.

3. Doddoli C, Aragon A, Barlesi F, et al. Does the extent of lymph node dissection influence outcome in patients with stage I non-small-cell lung cancer? Eur J Cardiothorac Surg. 2005;27:680-5.

4. Nakata M, Sawada S, Yamashita M, et al. Surgical treatments for multiple primary adenocarcinoma of the lung. Ann Thorac Surg. 2004;78:1194-9.

5. Ferguson MK, DeMeester TR, DesLauriers J, et al. Diagnosis and management of synchronous lung cancers. J Thorac Cardiovasc Surg. 1985;89:378-85.

6. Deschamps C, Pairolero PC, Trastek VF, et al. Multiple primary-lung cancers. Results of surgical treatment. J Thorac Cardiovasc Surg. 1990;99:769-77; discussion 777-8.

7. Martini N, Melamed MR. Multiple primary-lung cancers. J Thorac Cardiovasc Surg. 1975;70:606-12.

8. Paci M, Sgarbi G, Ferrari G, et al. Controversies over UICC-TNM classification of non-small cell lung cancer: model for a diagnostic path. Chest. 2002;122:754.

9. Deslauriers J, Gregoire J. Clinical and surgical staging of non-small cell lung cancer. Chest. 2000;117:96S-103S.

10. Vansteenkiste JF, De Belie B, Deneffe GJ, et al. Practical approach to patients presenting with multiple synchronous suspect lung lesions: a reflection on the current TNM classification based on 54 cases with complete follow-up. Lung Cancer. 2001;34:169-75.

11. Detterbeck FC, DeCamp MM Jr, Kohman LJ, et al. Lung cancer. Invasive staging: the guidelines. Chest. 2003;123:167S-75S.

12. Thomas $\mathrm{P}$, Doddoli $\mathrm{C}$, Thirion $\mathrm{X}$, et al. Stage I non-small cell lung cancer: a pragmatic approach to prognosis after complete resection. Ann Thorac Surg. 2002;73:1065-70.

13. Williams DE, Pairolero PC, Davis CS, et al. Survival of patients surgically treated for stage I lung cancer. J Thorac Cardiovasc Surg. 1981;82:70-6

14. Alexiou C, Beggs D, Onyeaka P, et al. Pneumonectomy for stage I (T1N0 and T2N0) nonsmall cell lung cancer has potent, adverse impact on survival. Ann Thorac Surg. 2003;76:1023-8.

15. Doddoli C, Barlesi F, Trousse D, et al. One hundred consecutive pneumonectomies after induction therapy for non-small cell lung cancer: an uncertain balance between risks and benefits. J Thorac Cardiovasc Surg. 2005;130:416-25.

16. Detterbeck FC, Jones DR, Kernstine KH, et al. Lung cancer. Special treatment issues. Chest. 2003;123:244S-58S.

17. Battafarano RJ, Meyers BF, Guthrie TJ, et al. Surgical resection of multifocal non-small cell lung cancer is associated with prolonged survival. Ann Thorac Surg. 2002;74:988-93; discussion 993-4.

18. Tung YW, Hsu CP, Shai SE, et al. Surgical feasibility of ipsilateral multifocal non-small cell lung cancer in different lobes: excellent survival in node-negative subgroup. Eur J Cardiothorac Surg. 2003; 24:1008-12. 
19. Tsunezuka Y, Matsumoto I, Tamura M, et al. The results of therapy for bilateral multiple primary-lung cancers: 30 years experience in a single centre. Eur J Surg Oncol. 2004;30:781-5.

20. Angeletti CA, Mussi A, Janni A, et al. Second primary-lung cancer and relapse: treatment and follow-up. Eur J Cardiothorac Surg. 1995;9: 607-11.

21. Adebonojo SA, Moritz DM, Danby CA. The results of modern surgical therapy for multiple primary-lung cancers. Chest. 1997;112:693-701.

22. Aziz TM, Saad RA, Glasser J, et al. The management of second primary-lung cancers. A single centre experience in 15 years. Eur J Cardiothorac Surg. 2002;21:527-33.

23. van Rens MT, Zanen P, Brutel de La Riviere A, et al. Survival in synchronous vs. single lung cancer: upstaging better reflects prognosis. Chest. 2000;118:952-8.
24. Ribet M, Dambron P. [Multiple primary cancers of the bronchi]. Ann Chir. 1993;47:721-8.

25. Birim O, Kappetein AP, Bogers AJ. Charlson comorbidity index as a predictor of long-term outcome after surgery for nonsmall cell lung cancer. Eur J Cardiothorac Surg. 2005;28:759-62.

26. Wright G, Manser RL, Byrnes G, et al. Surgery for non-small cell lung cancer: systematic review and meta-analysis of randomised controlled trials. Thorax. 2006;61:597-603.

27. Nakamura H, Kawasaki N, Taguchi M, et al. Survival following lobectomy vs limited resection for stage I lung cancer: a meta-analysis. Br J Cancer. 2005;92:1033-7.

28. Alam N, Darling G, Shepherd FA, et al. Postoperative chemotherapy in nonsmall cell lung cancer: a systematic review. Ann Thorac Surg. 2006;81:1926-36. 